\title{
División Alta del Nervio Fibular Superficial: Origen Precoz de los Nervios Cutáneos Dorsales del Pie
}

\author{
High Division of Superficial Fibular Nerve: Early Origin of the Dorsal Cutaneous Nerves of the Foot
}

"Olave, E.; ** Galaz, C. \& ** Retamal, P. \& *** Cruzat, C.

OLAVE, E.; GALAZ, C.; RETAMAL, P. \& CRUZAT, C. División alta del nervio fibular superficial: origen precoz de los nervios cutáneos dorsales del pie. Int. J. Morphol., 29(2):436-440, 2011.

RESUMEN: Los nervios cutáneos dorsales del pie se originan a partir del nervio fibular superficial (NFS), después que éste pasa desde el compartimiento lateral de la pierna a través de la fascia profunda, para luego distribuirse superficialmente en el dorso del pie. A veces, el nervio fibular superficial se divide precozmente, por lo que estos nervios se originan profundamente y con una disposición diferente. Con el propósito de dar a conocer en estos casos, los niveles de origen de los nervios cutáneos dorsales del pie y el punto de penetración en la fascia profunda de la pierna, se estudiaron 16 muestras de individuos, chilenos, adultos, fijadas en formaldehido al $10 \%$. En 2 de ellas, su origen se observó a un nivel alto, cerca de la parte proximal de la fíbula. En el primer caso, el nervio cutáneo dorsal intermedio se originó directamente de la división posterior del NFS; la división anterior del NFS se subdividió en dos ramos, los que después de un corto trayecto se unieron y formaron el nervio cutáneo dorsal medial. En el segundo caso, los nervios cutáneos dorsales intermedio y medial se originaron directamente del NFS, que después de un corto trayecto se dividió en estos dos nervios. El origen de los nervios cutáneos dorsales del pie se registró en relación al epicóndilo lateral del fémur y el paso de éstos a través de la fascia profunda de la pierna hacia su distribución cutánea fue relacionado al maléolo lateral. Aunque el origen de los nervios cutáneos dorsales del pie ocurra a un nivel próximo a la cabeza de la fíbula, perforan la fascia mencionada, en su tercio distal. Un conocimiento detallado del NFS y de sus ramos y variaciones anatómicas puede reducir lesiones iatrogénicas en éstos durante los procedimientos quirúrgicos realizados en el compartimiento lateral de la pierna.

PALABRAS CLAVE: Anatomía; Nervio fibular superficial; Pie; Nervios cutáneos dorsales.

\section{INTRODUCCIÓN}

Los nervios cutáneos dorsales del pie se originan a partir del nervio fibular superficial (NFS), cuando éste pasa desde el compartimiento lateral de la pierna a través de la fascia profunda, para distribuirse superficialmente en su dorso. El NFS es un ramo de la división del nervio fibular común (NFC), componente del nervio isquiático y que está destinado a inervar la musculatura de los compartimientos lateral y anterior y de la pierna así como al músculo extensor corto en el dorso del pie. Además otorga ramos sensitivos a las regiones anterolateral de la pierna y al dorso del pie.

El NFC y por ende, los ramos de su división, es susceptible de sufrir lesiones por la posición superficial en que se encuentra sobre el cuello de la fíbula. Es muy vulnerable en la exposición operatoria de la extremidad proximal de este hueso, pero también puede ser lesionado por vendajes y yesos apretados y en lesiones de la rodilla con aducción intensa (Healley \& Seybold, 1972). Lesiones directas como fracturas del cuello de la fíbula, laceraciones superficiales sobre este extremo del hueso y contusiones en la cara lateral de la rodilla, también es causa frecuente de lesiones del nervio a este nivel (Sunderland, 1985). Las afecciones mencionadas producen efectos negativos en la distribución motora y sensitiva del NFC y ramos de división.

El NFS inicialmente profundo al músculo fibular largo, envía ramos motores a los músculos fibulares largo y corto y en el tercio distal de la pierna perfora la fascia profunda, donde da origen a los nervios cutáneos dorsales intermedio y medial, los cuales se distribuyen por el dorso del pie.

\footnotetext{
* Facultad de Medicina, Universidad de La Frontera, Chile.

** Estudiantes de Medicina, Facultad de Medicina, Universidad Católica del Maule, Chile

*** Facultad de Medicina, Universidad Católica del Maule, Chile
} 
Sin embargo, esta descripción encontrada en diversos textos de Anatomía (Testut \& Latarjet, 1969; Williams et al., 1995; Moore \& Dalley, 2002), puede presentar variaciones, de tal manera de observar padrones distintos en la formación de estos nervios cutáneos.

Este trabajo presenta casos de división alta del nervio fibular superficial, mostrando el origen de los nervios cutáneos dorsales del pie a un nivel próximo al extremo proximal de la fíbula, los cuales, independiente de su origen, atraviesan la fascia profunda de la pierna, en su tercio distal.

\section{MATERIAL Y METODO}

Para el estudio se utilizaron 16 piernas, 9 izquierdas y 7 derechas, de individuos adultos, chilenos, fijadas en formaldehído al $10 \%$, las cuales son parte del Laboratorio de Anatomía de la Facultad de Medicina de la Universidad Católica del Maule, Talca.

Inicialmente, se localizó la parte más prominente del epicóndilo lateral del fémur (EPCL), así como también la parte prominente del maléolo lateral (ML). Ambos se utilizaron como puntos de referencia para ubicar los niveles de origen de los nervios cutáneos y punto de perforación en la fascia. Se realizó disección por planos, alcanzando el nervio fibular común así como su punto de división en nervios fibular superficial y profundo. Se identificó los ramos cutáneos dorsales de origen alto, donde se midió con un caliper digital Mitutoyo, de precisión 0,05 mm, el nivel de origen de ellos respecto al EPCL. A nivel del punto de perforación en el tercio distal de la pierna, se registró la distancia entre este punto y el ML.

De todas las muestras se realizaron esquemas y se fotografiaron las más representativas.

\section{RESULTADOS}

De las 16 muestras observadas, en 2 de ellas su origen se observó a un nivel alto, cerca de la parte proximal de la fíbula.

En el primer caso, el nivel de división del NFC en NFS y NF profundo se registró a 69,2 mm distal al EPCL y la división del NFS en dos nervios cutáneos dorsales del pie a $72,7 \mathrm{~mm}$ distal al EPCL.

El nervio cutáneo dorsal intermedio del pie se originó directamente de la división posterior del NFS, de corta longitud; la división anterior del NFS se subdividió en dos ramos, los que después de un corto trayecto se unieron a 127,2 mm distal al EPCL y formaron el nervio cutáneo dorsal medial del pie (Fig. 1).

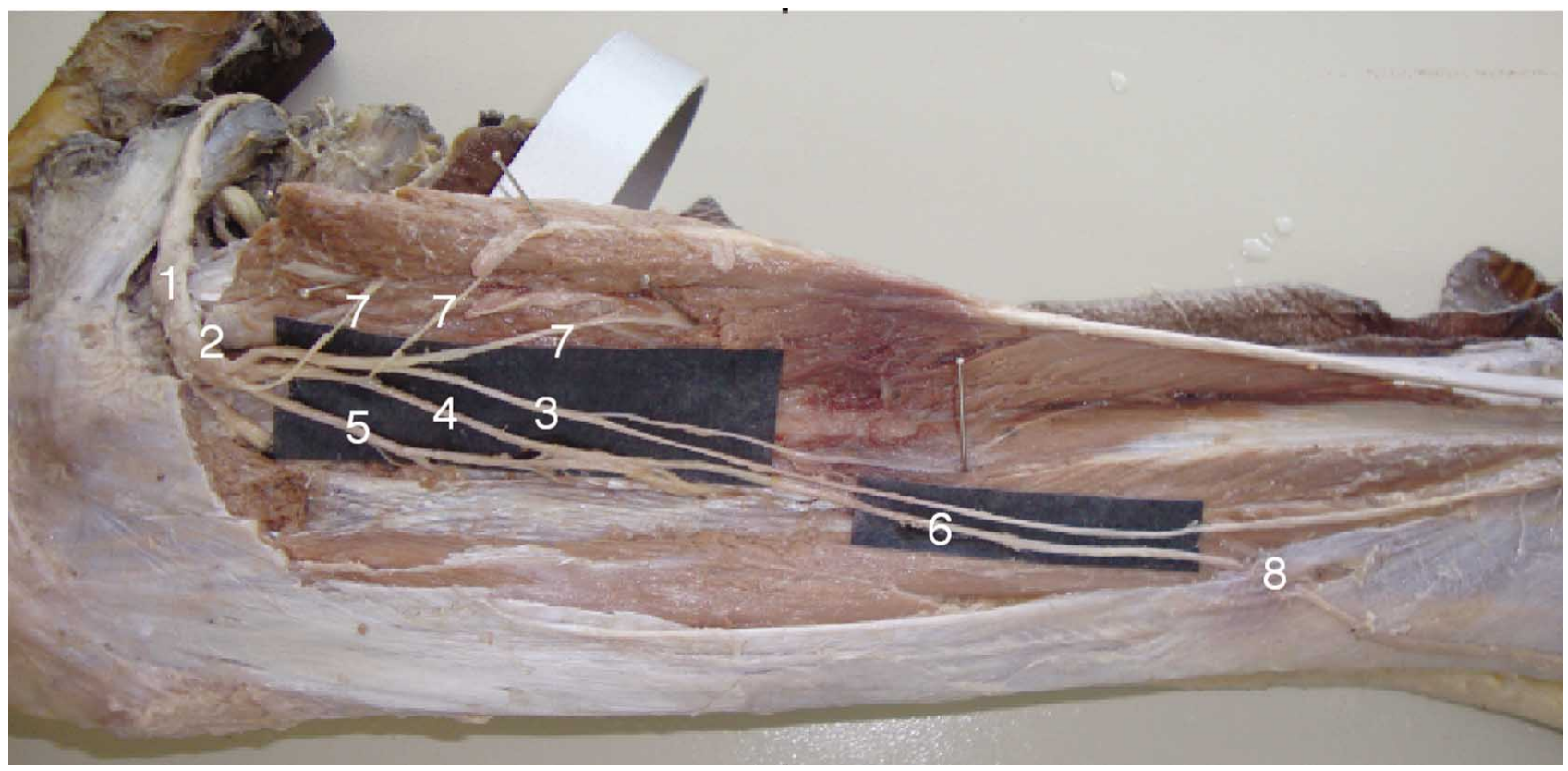

Fig. 1. 1. Nervio fibular común; 2. Nervio fibular superficial; 3. Nervio cutáneo dorsal intermedio del pie; 4 y 5. Ramos de formación del nervio cutáneo dorsal medial del pie; 6. Nervio cutáneo dorsal medial del pie; 7. Ramos para el músculo fibular largo; 8 . Punto de perforación de la fascia profunda de la pierna por el nervio cutáneo dorsal medial del pie. 
El punto de perforación en la fascia se registró a 37,0 $\mathrm{mm}$ proximal al ML para el caso del nervio cutáneo dorsal intermedio y de 126,0 mm proximal al ML para el caso del nervio cutáneo dorsal medial.

En el segundo caso, los nervios cutáneos dorsales intermedio y medial se originaron directamente del NFS, que después de un corto trayecto se dividió en estos dos nervios. El nivel de división del NFC se registró a 53,6 mm del EPCL; la división del NFS en dos nervios cutáneos dorsales del pie ocurrió a $84,7 \mathrm{~mm}$ distal al EPCL y el punto de perforación de la fascia, punto común para ambos nervios se registró a $104 \mathrm{~mm}$ proximal al ML.

\section{DISCUSIÓN}

El nervio fibular superficial es inicialmente profundo al músculo fibular largo y envía ramos motores a los músculos fibulares largo y corto; en el tercio distal de la pierna perfora la fascia profunda, donde se divide en ramos medial y lateral. El ramo medial pasa anteriormente al tobillo y se divide en dos nervios digitales dorsales, uno alcanzando el lado medial del hallux y el otro, los lados adyacentes del segundo y tercer dedo. El ramo lateral, atraviesa el dorso del pie lateralmente, dividiéndose en ramos digitales dorsales que alcanzan los lados adyacentes del tercero al quinto dedo. La parte lateral del quinto dedo y lados adyacentes del hallux y del segundo dedo son inervados, el primero por el nervio sural y los segundos por el ramo terminal del nervio fibular profundo. Con frecuencia, algunos de los ramos laterales del nervio fibular superficial están ausentes y son substituidos por ramos del nervio sural (Williams et al.).

El conocimiento del trayecto y distribución del NFS es de gran importancia para la cirugía de la parte proximal y media del compartimiento lateral de la pierna, lo que incluye conocer sus variaciones más frecuentes y así evitar lesiones durante procedimientos quirúrgicos en el área. Entre estos últimos tenemos colgajos fasciocutáneos, fasciotomías, anestesia de la región del tobillo, síndromes dolorosos traumáticos, etc. (Canovas et al., 1996).

Dentro de las variaciones del NFS se ha descrito la división alta de éste, donde los ramos de división, ramos cutáneos dorsales del pie, medial e intermedio, pueden tener un trayecto en el mismo compartimiento lateral (Browne \& Morris, 2007) o en compartimientos separados, lateral y anterior, (Solomon et al., 2001; Nagabhooshana et al., 2009).

Una característica interesante es la formación del nervio cutáneo dorsal medial en uno de nuestros casos, donde la división anterior del NFS se subdividió en dos ramos, los que después de un corto trayecto se unieron y formaron a este nervio, disposición no encontrada en la literatura consultada.

La distribución del NFS ha sido padronizada por Ucerler \& Ikiz (2005), los que determinaron 3 padrones. El tipo I $(63,3 \%)$ es el clásico descrito en los diversos textos de Anatomía, donde el tronco del nervio perfora la fascia profunda de la pierna y se divide en los nervios cutáneos dorsales medial e intermedio del pie; el tipo $2(26,7 \%)$ corresponde a un origen independiente de estos nervios cutáneos a partir del NFS, que es la disposición encontrada en los casos de nuestra serie, presentándose en un porcentaje menor $(12,5 \%)$. En el tipo 3 (10\%), el NFS perforó independiente la fascia profunda y tuvo luego, un trayecto semejante al nervio cutáneo dorsal medial del pie.

Un porcentaje similar al de Ucerler \& Ikiz fue observado por Kurtoglu et al. (2006), donde la división del NFS en dos ramos antes de perforar la fascia se encontró en $27,5 \%$. El estudio de estos últimos fue realizado en 20 fetos. Otros autores que encontraron porcentaje similar al tipo 2 de Ucerler \& Ikiz, fueron Agthong et al. (2008) que estudiaron 85 piernas y esta disposición se presentó en $28,2 \%$. Un resultado menor (4,5\%) había sido relatado por Aigner et al. (2004) quienes investigaron 111 piernas.

En disección de 5 individuos, Canella et al. (2009) señalaron que en uno de ellos (20\%) el NFS tuvo una división más proximal, antes de pasar a través de la fascia profunda de la pierna. Sin embargo, también realizaron el estudio en 30 voluntarios con técnica de sonografía y encontraron sólo un 6,7\% en que el NFS se dividía antes de perforar la fascia.

Con relación al punto de perforación de la fascia profunda de la pierna por parte del NFS como tronco único, Agthong et al. señalaron que en $71,8 \%$ de las muestras que estudiaron, la distancia entre el punto de perforación del NFS respecto a la línea intermaleolar fue en promedio de $7,7 \mathrm{~cm}$. Por su parte, Canella et al. reportaron un promedio de $9,22 \mathrm{~cm}$.

Cuando los ramos cutáneos dorsales medial e intermedio del pie, se originan a partir de una división alta del NFS, se han informado valores variables de la distancia en que ocurre ese paso a través de la fascia, respecto a los maléolos. En el caso presentado por Browne \& Morris, el nervio cutáneo dorsal medial perforó la fascia profunda 13 $\mathrm{cm}$ proximal al maléolo lateral y el nervio cutáneo dorsal intermedio a $11,5 \mathrm{~cm}$ proximal al maléolo. En una de nuestras muestras, el nervio cutáneo dorsal medial pasó a través de la fascia a la misma distancia informada por estos autores. El otro nervio, intermedio, estuvo muy cerca del maléolo 
lateral, por lo tanto, no relacionado con el valor informado por Browne \& Morris, pero un valor semejante a estos últimos, también fue registrado por Nagabhooshana et al. En el segundo caso presentado en este trabajo, las distancias obtenidas para ambos ramos, que pasaron a través de la fascia al mismo nivel, estuvieron cercanas a las de los autores mencionados.
De acuerdo a lo observado en este estudio y a la información recopilada de la literatura consultada, hay variaciones en la disposición de los ramos del NFS y niveles de perforación de la fascia profunda, por lo que es importante considerar estas variables durante los procedimientos quirúrgicos efectuados en la región lateral de la pierna y así evitar lesiones en los nervios aludidos.

OLAVE, E.; GAlAZ, C.; RETAMAL, P. \& CRUZAT, C. High division of superficial fibular nerve: Early origin of the dorsal cutaneous nerves of the foot. Int. J. Morphol., 29(2):436-440, 2011.

SUMMARY: The dorsal cutaneous nerves of the foot originate from the superficial fibular nerve (SFN), then pass from the lateral compartment of the leg piercing the deep fascia, thus distributed superficially in the dorsum of the foot. Sometimes SFN splits early, so these nerves originate deeply and with a different arrangement. The objective of this research was to study in these cases, the origin of the dorsal cutaneous nerves of the foot and pierce point level in the deep fascia of the leg. We studied 16 formolized samples of Chilean adult individuals. In two of them its origin was observed at a high level, near the proximal epiphysis of the fibula. In the first case the intermediate dorsal cutaneous nerve arose directly from the posterior division of SFN, of short course; the anterior division of SFN was subdivided into two branches, which after a short course, both branches joined to form the medial dorsal cutaneous nerve. In the second case the medial and intermediate dorsal cutaneous nerves originated directly from the SFN, which after a short course, it divided in these two nerves. The origin level was recorded in relation to the lateral epicondyle of the femur and the passage of these nerves through the deep fascia of the leg for its skin distribution was located in relation to the lateral malleolus. Although the origin of the dorsal cutaneous nerves of the foot occur very close to the proximal epiphysis of the fibula, passing through the mentioned fascia in its distal third. A detailed knowledge of the branches of the SFN and variations can reduce injuries in these nerves during surgical procedures in the lateral compartment of the leg.

KEY WORDS: Anatomy; Superficial fibular nerve; Foot; Dorsal cutaneous nerves.

\section{REFERENCIAS BIBLIOGRÁFICAS}

Aigner, F.; Longato, S.; Gardetto, A.; Deibl, M.; Frtisch, H. \& Piza-Katzer, H. Anatomic survey of the common fibular nerve and its branching patterns with regard to the intermuscular septa of the leg. Clin. Anat., 17:50312, 2004.

Agthong, S.; Huanmanop, T.; Sasivongsbhakdi, T. Ruenkhwan, K.; Piyawacharapun, A. \& Chentanez,V. Anatomy of the superficial peroneal nerve related to the harvesting for nerve graft. Surg. Radiol. Anat., 30:1458,2008 .

Browne,J. \& Morris, M. Variant superficial fibular (peroneal) nerve anatomy in the middle third of the lateral leg. Clin. Anat., 20:996-7, 2007.

Canella, C.; Demondion, X.; Guillin, R.; Boutry, N.; Peltier, J. \& Cotton, A. Anatomic study of the superficial peroneal nerve using sonography. AMR, 193:174-9, 2009.

Canovas, F.; Bonnel, F. \& Kouloumdjian, P. The superficial peroneal nerve at the foot. Organisation, surgical applications. Surg. Radiol. Anat., 18:241-4, 1996.
Healley, J. \& Seybold, W. Anatomía Clínica. México, Interamericana, 1972.

Kurtoglu, Z.; Aktekin, M. \& Haluk Uluutku,M. Branching patterns of the common and superficial fibular nerves in fetus. Clin. Anat., 19:621-26, 2006.

Moore, Kl. \& Dalley, AF. Anatomía con orientación clínica. $4^{\mathrm{a}}$ Ed. Buenos Aires, Panamericana, 2002.

Nagabhooshana; S.; Ramana Vollala, V.; Rodrigues, V. \& Rao, M. Anomalous superficial peroneal nerve and variant cutaneous innervation of the sural nerve on the dorsum of the foot: a case report. Cases Journal, 2:197, 2009.

Solomon, L.; Ferris, R.; Tedman, R. \& Henneberg, M. Surgical anatomy of the sural and superficial fibular nerves with and emphasis on the approach to the lateral malleolus. J. Anat., 199:717-23, 2001.

Sunderland, S. Nervios Periféricos y sus lesiones. Barcelona, Salvat, 1985. 
OLAVE, E.; GALAZ, C.; RETAMAL, P. \& CRUZAT, C. División alta del nervio fibular superficial: origen precoz de los nervios cutáneos dorsales del pie. Int. J. Morphol., 29(2):436-440, 2011.

Testut, L. \& Latarjet, A. Tratado de Anatomía Humana. Barcelona, Salvat, 1969. V. 3.

Ucerler, H. \& Ikiz, A. The variation of the sensory branches of the superficial peroneal nerve course and its clinical importance. Foot Ankle Int. 26(11): 942-6, 2005.

Williams, R L.; Warwick, R.; Dyson, M. \& Bannister, L. H. Gray Anatomia. 37 ${ }^{\mathrm{a}}$ Ed. Rio de Janeiro, GuanabaraKoogan, 1995. V. 2.
Dirección para correspondencia:

Prof. Dr. Enrique Olave

Facultad de Medicina

Uiversidad de La FRontera

Casilla 54-D

Temuco

CHILE

Email: eolave@ufro.cl

Recibido : 27-12-2010

Aprobado: 12-03-2011 